\title{
Morrey Spaces for Nonhomogeneous Metric Measure Spaces
}

\author{
Cao Yonghui and Zhou Jiang \\ Department of Mathematics, Xinjiang University, Urumqi 830046, China \\ Correspondence should be addressed to Zhou Jiang; zhoujiangshuxue@126.com
}

Received 6 May 2013; Accepted 14 August 2013

Academic Editor: Vakhtang M. Kokilashvili

Copyright (C) 2013 C. Yonghui and Z. Jiang. This is an open access article distributed under the Creative Commons Attribution License, which permits unrestricted use, distribution, and reproduction in any medium, provided the original work is properly cited.

The authors give a definition of Morrey spaces for nonhomogeneous metric measure spaces and investigate the boundedness of some classical operators including maximal operator, fractional integral operator, and Marcinkiewicz integral operators.

\section{Introduction}

During the past fifteen years, many results from real and harmonic analysis on the classical Euclidean spaces have been extended to the spaces with nondoubling measures only satisfying the polynomial growth condition (see [19]). The Radon measure $\mu$ on $\mathbb{R}^{d}$ is said to only satisfy the polynomial growth condition, if there exists a positive constant $c$ such that for all $x \in \mathbb{R}^{d}$ and $r>0, \mu(B(x, r)) \leq$ $c r^{n}$, where $n$ is some fixed number in $(0, d]$ and $B(x, r)=$ $\left\{y \in \mathbb{R}^{d}:|x-y|<r\right\}$. The analysis associated with such nondoubling measures $\mu$ is proved to play a striking role in solving the long-standing open Painlevés problem by Tolsa [6]. Obviously, the nondoubling measure $\mu$ with the polynomial growth condition may not satisfy the wellknown doubling condition, which is a key assumption in harmonic analysis on spaces of homogeneous type. To unify both spaces of homogeneous type and due to the fact that the metric spaces endow with measures only satisfying the polynomial growth condition, Hytönen [10] introduced a new class of metric measure spaces satisfying both the so-called geometrically doubling and the upper doubling conditions (see Definition 3), which are called nonhomogeneous spaces. Recently, many classical results have been proved still valid if the underlying spaces are replaced by the nonhomogeneous spaces of Hytönen (see [11-17]).

In this paper, we give a natural definition of Morrey spaces associated with the nonhomogeneous spaces of Hytönen and investigate the boundedness of some classical operators including maximal operator, fractional integral operator and
Marcinkiewicz integrals operator. To state the main results of this paper, we first recall some necessary notion, and notations. The following notions of geometrically doubling and upper doubling metric measure spaces were originally introduced by Hytönen [10].

Definition 1. A metric space $(\mathscr{X}, d)$ is said to be geometrically doubling if there exists some $N_{0} \in \mathbb{N}$ such that, for any ball $B(x, r) \subset \mathcal{X}$, there exists a finite ball covering $\left\{B\left(x_{i}, r / 2\right)\right\}_{i}$ of $B(x, r)$ such that the cardinality of this covering is at most $N_{0}$.

Remark 2. Let $(\mathscr{X}, d)$ be a metric space. In [10], Hytönen showed that the following statements are mutually equivalent.

(1) $(\mathscr{X}, d)$ is geometrically doubling.

(2) For any $\varepsilon \in(0,1)$ and any ball $B(x, r) \subset \mathscr{X}$, there exists a finite ball covering $\left\{B\left(x_{i}, \varepsilon r\right)\right\}_{i}$ of $B(x, r)$ such that the cardinality of this covering is at most $N_{0} \varepsilon^{-n}$, where $n=\log _{2} N_{0}$.

(3) For any $\varepsilon \in(0,1)$ and any ball $B(x, r) \subset \mathscr{X}$ contains at most $N_{0} \varepsilon^{-n}$ centers $\left\{x_{i}\right\}_{i}$ of disjoint balls $\left\{B\left(x_{i}, \varepsilon r\right)\right\}_{i}$.

(4) There exists $M \in \mathbb{N}$ such that any ball $B(x, r) \subset$ $\mathscr{X}$ contains at most $M$ centers $\left\{x_{i}\right\}_{i}$ of disjoint balls $\left\{B\left(x_{i}, r / 4\right)\right\}_{i=1}^{M}$.

Definition 3. A metric measure space $(\mathscr{X}, d, \mu)$ is said to be upper doubling if $\mu$ is a Borel measure on $\mathscr{X}$ and there exist a dominating function $\lambda: \mathscr{X} \times(0, \infty) \rightarrow(0, \infty)$ and a 
positive constant $c_{\lambda}$ such that, for each $x \in \mathscr{X}, r \rightarrow \lambda(x, r)$ is nondecreasing and

$$
\mu(B(x, r)) \leq \lambda(x, r) \leq c_{\lambda} \lambda\left(x, \frac{r}{2}\right) \quad \forall x \in \mathscr{X}, r>0 .
$$

It was proved in [14] that there exists a dominating function $\tilde{\lambda}$ related to $\lambda$ satisfying the property that there exists a positive constant $c_{\tilde{\lambda}}$ such that $\tilde{\lambda} \leq \lambda, c_{\tilde{\lambda}} \leq c_{\lambda}$, and, for all $x, y \in \mathscr{X}, r>0$ with $d(x, y) \leq r, \widetilde{\lambda}(x, r) \leq c_{\tilde{\lambda}} \tilde{\lambda}(y, r)$. Based on this, in this paper, we always assume that the dominating function $\lambda$ also satisfies it.

The following coefficients $\delta(B, S)$ for all ball $B$ and $S$ were introduced in [10] as analogues of Tolsa's number $K_{Q, R}$ in [5].

Definition 4. For all balls $B \subset S$, let

$$
\delta(B, S)=\int_{(2 S-B)} \frac{d \mu(x)}{\lambda\left(c_{B}, d\left(x, c_{B}\right)\right)},
$$

where, as in the above mentioned, and in what follows, for a ball $B=B\left(c_{B}, r_{B}\right)$ and $\rho>0, \rho B=B\left(c_{B}, \rho r_{B}\right)$.

Definition 5. Let $\alpha, \beta \in(0, \infty)$. A ball $B \subset \mathscr{X}$ is called $(\alpha, \beta)$ doubling if $\mu(\alpha B) \leq \beta \mu(B)$.

It was proved in [10] that if a metric measure space $(\mathscr{X}, d, \mu)$ is upper doubling and $\alpha, \beta \in(0, \infty)$ satisfying $\beta>c_{\lambda}^{\log _{2} \alpha}=\alpha^{v}$, then, for any ball $B$, there exists some $j \in \mathbb{N} \cup\{0\}$ such that $\alpha^{j} B$ is $(\alpha, \beta)$-doubling. Moreover, let $(\mathscr{X}, d, \mu)$ be geometrically doubling, $\beta>\alpha^{n}$ with $n=\log _{2} N_{0}$ and $\mu$ a Borel measure on $\mathscr{X}$ which is finite on bounded sets. Hytönen [10] also showed that, for $\mu$-almost every $x \in \mathscr{X}$, there exist arbitrary small $(\alpha, \beta)$-doubling balls centered at $x$. Furthermore, the radii of these balls may be chosen to be from $\alpha^{-j} B$ for $j \in \mathbb{N}$ and any preassigned number $r>0$. Throughout this paper, for any $\alpha \in(1, \infty)$ and ball $B$, the smallest $\left(\alpha, \beta_{\alpha}\right)$-doubling ball of the form $\alpha^{j} B$ with $j \in \mathbb{N}$ is denoted by $\widetilde{B}^{\alpha}$, where

$$
\beta_{\alpha}=\max \left\{\alpha^{3 n}, \alpha^{3 v}\right\}+30^{n}+30^{v} .
$$

In what follows, by a doubling ball we mean a $\left(6, \beta_{6}\right)$ doubling ball and $\widetilde{B}^{6}$ is simply denoted by $\widetilde{B}$.

Let $k>1$ and $1 \leq q \leq p<\infty$. We define the Morrey space $M_{q}^{p}(k, \mu)$ associated with the nonhomogeneous spaces of Hytönen. This is an analogy of [18-20].

Definition 6. Let $k>1$ and $1 \leq q \leq p<\infty$, as

$$
M_{q}^{p}(k, \mu)=\left\{f \in L_{\mathrm{loc}}^{q}:\|f\|_{M_{q}^{p}(k, \mu)}<\infty\right\},
$$

where

$$
\|f\|_{M_{q}^{p}(k, \mu)}=\sup _{B} \mu(k B)^{1 / p-1 / q}\left(\int_{B}|f|^{q} d \mu\right)^{1 / q} .
$$

Clearly we have $L^{p}(\mu)=M_{p}^{p}(k, \mu)$ and $M_{q_{1}}^{p} \subset M_{q_{2}}^{p}$, $1 \leq q_{2} \leq q_{1} \leq p$. If the underlying spaces are replaced by the nonhomogeneous spaces of Tolsa or Euclidean spaces, the definition of Morrey spaces can been seen in [18]. We will prove in Section 2 that the Morrey space is independent of choice of $k$.

In [21], Chiarenza and Frasca showed that the HardyLittlewood maximal operator is bounded on the Morrey space. By establishing a pointwise estimate of fractional integrals in terms of the maximal function, they also showed the boundedness of fractional integral operator on Morrey space. If the underlying spaces are replaced by the nonhomogeneous spaces of Tolsa, Sawano and Tanaka also obtained these results in [18]. When the underlying spaces are the nonhomogeneous spaces of Hytönen, these operators have been discussed in Lebesgue space and RBMO space (see $[22,23])$.

Main theorems of this paper are stated in each section. The definition of Morrey space and its equivalent definition are shown in Section 2. Section 3 is devoted to the study of maximal operator and fractional maximal operator. Section 4 deals with the fractional integral operator for the nonhomogeneous spaces of Hytönen. In Section 5, we investigate the behavior of the Marcinkiewicz integrals operator. In what follows the letter $c$ will be used to denote constants that may change from one occurrence to another.

\section{Morrey Space and Its Equivalent Definition}

We firstly prove that the definition of Morrey space is independent of the choice of the parameter $k$ (see $[18$, Proposition 1.1]).

Theorem 7. Let $k, s>1$; then $M_{q}^{p}(k, \mu) \approx M_{q}^{p}(s, \mu)$.

Proof. This result is a special case of the results in [24, Theorem 1.2]. For the sake of convenience, we provide the details. Let $k \leq s$. By the definition of Morrey space, we have

$$
\begin{aligned}
\|f\|_{M_{q}^{p}(s, \mu)} & =\sup _{B} \mu(s B)^{1 / p-1 / q}\left(\int_{B}|f| d \mu\right)^{1 / q} \\
& \leq \sup _{B} \mu(k B)^{1 / p-1 / q}\left(\int_{B}|f| d \mu\right)^{1 / q} \\
& =\|f\|_{M_{q}^{p}(k, \mu)},
\end{aligned}
$$

where $1 / p-1 / q<0$. So the inclusion $M_{q}^{p}(k, \mu) \subset M_{q}^{p}(s, \mu)$ is obvious.

Let $f \in M_{q}^{p}(s, \mu)$ and ball $B \subset \mathscr{X}$. Exploiting Remark 2(2), where $\varepsilon=(k-1) / s$, we have that there exists ball $B_{1}, B_{2}, \ldots, B_{N}$ with the same radius $r=\varepsilon r_{B}$ such that

$$
\begin{gathered}
B \subset \cup_{i=1}^{N} B_{i}, \quad s B_{i} \subset k B \\
(i=1,2, \ldots, N), \quad N \leq N_{0}\left(\frac{s}{k-1}\right)^{n} .
\end{gathered}
$$


Using this covering, we obtain

$$
\begin{aligned}
\mu(k B)^{1 / p-1 / q}\left(\int_{B}|f|^{q} d \mu\right)^{1 / q} \\
\leq \sum_{i=1}^{N} \mu(k B)^{1 / p-1 / q}\left(\int_{B_{i}}|f|^{q} d \mu\right)^{1 / q} \\
\leq \sum_{i=1}^{N} \mu\left(s B_{i}\right)^{1 / p-1 / q}\left(\int_{B_{i}}|f|^{q} d \mu\right)^{1 / q} \\
\leq N_{0}\left(\frac{s}{k-1}\right)^{n}\|f\|_{M_{q}^{p}(s, \mu)} .
\end{aligned}
$$

That is, $\|f\|_{M_{q}^{p}(k, \mu)} \leq c\|f\|_{M_{q}^{p}(s, \mu)}$. We complete the proof of the theorem.

With this theorem in mind, we sometimes omit parameter $k$ in $M_{q}^{p}(k, \mu)$.

Let $Q \stackrel{q}{=}\left\{B \subset \mathscr{X}: B\right.$ is $\left(6, \beta_{6}\right)$-doubling ball $\}$. Now we give an equivalent definition of Morrey space.

Definition 8 . Let $1 \leq q \leq p<\infty$; as

$$
M_{q}^{p}(d)=\left\{f \in L_{\mathrm{loc}}^{q}:\|f\|_{M_{q}^{p}(d)}<\infty\right\}
$$

where

$$
\|f\|_{M_{q}^{p}(d)}=\sup _{B \in Q} \mu(B)^{1 / p-1 / q}\left(\int_{B}|f|^{q} d \mu\right)^{1 / q} .
$$

This definition and Theorem 9 are analogy of [20].

Theorem 9. Let $1 \leq q<p<\infty$ and $\beta_{6}>\left(6^{n}\right)^{q p /(p-q)}$; then $M_{q}^{p}(d) \approx M_{q}^{p}(\mu)$.

Proof. We only need to prove that $\|f\|_{M_{q}^{p}(\mu)} \leq c\|f\|_{M_{q}^{p}(d)}$.

For every ball $B_{0}=B\left(c_{B}, r_{0}\right)$ and $x \in B_{0}$, let $B\left(x, 6^{-i_{x}} r_{0}\right)$ be the largest doubling ball centered at $x$, having radius $6^{-i_{x}} r_{0}, i_{x} \in \mathbb{N}$. So $B\left(c_{B}, r_{0}\right) \subset \cup_{x \in B_{0}} B\left(x, 6^{-i_{x}} r_{0}\right)$. By Besicovitch covering lemma, there is a subcollection $A=$ $\left\{B\left(x_{i}, 6^{-j_{x_{i}}} r_{0}\right)\right\}_{i=1}^{\infty}$ that covers $B\left(c_{B}, r_{0}\right)$ so that no point belongs to more than $c_{X}$ of $\left\{B\left(x_{i}, 6^{-j_{x_{i}}} r_{0}\right)\right\}_{i=1}^{\infty}$, where $c_{X}$ only depends on space $\mathscr{X}$. We write $A_{i}=\left\{B \in A: r_{B}=6^{-i} r_{0}\right\}$. Using Remark 2(2), we know, cardinal number of set $A_{i} \leq N_{0} 6^{\text {in }}$. For all $B\left(x, 6^{-i} r_{0}\right) \in A_{i}$, we have

$$
\mu\left(6 B_{0}\right) \geq \mu\left(B\left(x, r_{0}\right)\right) \geq \beta_{6}^{i} \mu\left(B\left(x, 6^{-i} r_{0}\right)\right) .
$$

So

$$
\begin{aligned}
& \mu\left(6 B_{0}\right)^{1 / p-1 / q}\left(\int_{B_{0}}|f|^{q} d \mu\right)^{1 / q} \\
& \leq \sum_{i=1}^{\infty} \sum_{B \in A_{i}} \mu\left(6 B_{0}\right)^{1 / p-1 / q}\left(\int_{B}|f|^{q} d \mu\right)^{1 / q}
\end{aligned}
$$

$$
\begin{aligned}
& \leq \sum_{i=1}^{\infty} \sum_{B \in A_{i}}\left(\beta_{6}^{i}\right)^{1 / p-1 / q}\left(\mu\left(B\left(x, 6^{-i} r_{0}\right)\right)\right)^{1 / p-1 / q} \\
& \quad \times\left(\int_{B\left(x, 6^{-i} r_{0}\right)}|f|^{q} d \mu\right)^{1 / q} \\
& \leq c\|f\|_{M_{q}^{p}(d)} \sum_{i=1}^{\infty} N_{0} 6^{i n}\left(\beta_{6}^{i}\right)^{1 / p-1 / q} \\
& \leq c\|f\|_{M_{q}^{p}(d)} .
\end{aligned}
$$

\section{Maximal Inequalities}

In this section we will investigate some maximal inequalities. Now we give the definitions of some maximal operators.

Definition 10. Let $\rho>0, r>1, \alpha \in(0,1)$, as

$$
\begin{gathered}
M_{\rho} f(x)=\sup _{x \in B} \frac{1}{\mu(\rho B)} \int_{B}|f| d \mu, \\
M_{\rho}^{\alpha} f(x)=\sup _{x \in B} \frac{1}{\mu(\rho B)^{1-\alpha}} \int_{B}|f| d \mu,
\end{gathered}
$$

$$
\begin{aligned}
& M_{r, \rho} f(x)=\sup _{x \in B}\left[\frac{1}{\mu(\rho B)} \int_{B}|f|^{r} d \mu\right]^{1 / r}, \\
& M_{r, \rho}^{\alpha} f(x)=\sup _{x \in B}\left[\frac{1}{\mu(\rho B)^{1-\alpha r}} \int_{B}|f|^{r} d \mu\right]^{1 / r} .
\end{aligned}
$$

In $[11,22,25-27]$, the boundedness of these maximal operators has been proven in Lebesgue spaces.

Lemma 11. Let $p>1, \rho>0$. Then the maximal operators $M_{\rho}$ and $M_{r, \rho}$ are bounded on $L^{p}(\mu)$ space.

Lemma 12. Let $\alpha \in(0,1), 1<r<p<1 / \alpha, \rho \geq 5$, and $1 / q=1 / p-\alpha$. Then the maximal operator $M_{r, \rho}^{\alpha}$ is bounded from $L^{p}(\mu)$ space to $L^{q}(\mu)$ space.

Remark 13. When $r=1$, Lemma 11 also is right.

Now we extend these results to the Morrey spaces.

Theorem 14. If $\rho>1$ and $1<r<q \leq p<\infty$, then the maximal operators $M_{\rho}$ and $M_{r, \rho}$ are bounded on $M_{q}^{p}(\mu)$ space.

Proof. The proof of the boundedness of $M_{\rho}$ has been obtained in $[24,28]$. We only prove the boundedness of $M_{r, \rho}$. For simplicity, we take $\rho=2$. Let $B_{0}=B\left(x_{0}, r_{0}\right)$ and $f=f_{1}+f_{2}$, where $f_{1}(x)=f(x) \chi_{9 B_{0}}(x)$. Then for every $y \in B_{0}$ we have

$$
M_{r, \rho} f(y) \leq M_{r, \rho} f_{1}(y)+M_{r, \rho} f_{2}(y) .
$$

From the definitions of $M_{r, \rho}$ and $f_{2}$ it follows that

$$
M_{r, \rho} f_{2}(y) \leq \sup _{y \in B, r_{B} \geq 8 r_{0}}\left[\frac{1}{\mu(2 B)} \int_{B}|f|^{r} d \mu\right]^{1 / r} .
$$


For $y \in B_{0} \cap B, r_{B} \geq 8 r_{0}$, the simple calculus yields $B_{0} \subset$ $(3 / 2) B$. Thus we have

$$
M_{r, \rho} f_{2}(y) \leq \sup _{y \in B_{0} \subset B}\left[\frac{1}{\mu((4 / 3) B)} \int_{B}|f|^{r} d \mu\right]^{1 / r}
$$

It follows that

$$
\begin{aligned}
& \mu\left(12 B_{0}\right)^{1 / p-1 / q}\left(\int_{B_{0}}|M r, \rho f|^{q} d \mu\right)^{1 / q} \\
& \leq \mu\left(12 B_{0}\right)^{1 / p-1 / q}\left(\int_{B_{0}}\left|M_{r, \rho} f_{1}\right|^{q} d \mu\right)^{1 / q} \\
&+\mu\left(12 B_{0}\right)^{1 / p-1 / q}\left(\int_{B_{0}}\left|M_{r, \rho} f_{2}\right|^{q} d \mu\right)^{1 / q} \\
& \leq \mu\left(12 B_{0}\right)^{1 / p-1 / q}\left(\int_{X}\left|M_{r, \rho} f_{1}\right|^{q} d \mu\right)^{1 / q} \\
&+\mu\left(B_{0}\right)^{1 / p-1 / q}\left(\int_{B_{0}}\left|M_{r, \rho} f_{2}\right|^{q} d \mu\right)^{1 / q} \\
& \leq c\|f\|_{M_{q}^{p}(4 / 3, \mu) \cdot} \\
& \leq c \mu\left(12 B_{0}\right)^{1 / p-1 / q}\left(\int_{X}\left|f_{1}\right|^{q} d \mu\right)^{1 / q} \\
& \leq+\left.\|f\|_{M_{q}^{p}(4 / 3, \mu)}\right|_{y \in B_{0} p(4 / 3, \mu)} \sup _{y \in B_{0} \subset B} \mu(B)^{1 / p+1 / r-1 / q} \mu\left(\frac{4}{3} B\right)^{1 / q-1 / p-1 / r} \\
&+\mu\left(B_{0}\right)^{1 / p} \sup _{y \in B_{0} \subset B}\left(\frac{1}{\mu((4 / 3) B)} \int_{B}|f|^{r} d \mu\right)^{1 / p-1 / q}\left(\int_{9 B_{0}}|f|^{q} d \mu\right)^{1 / q} \\
&+\mu\left(B_{0}\right)^{1 / p} \sup _{y \in B_{0} \subset B} \mu\left(\frac{4}{3} B\right)^{-1 / r} \mu(B)^{1 / r-1 / q}\left(\int_{B}|f|^{q} d \mu\right)^{1 / q} \\
& \leq c \mu\left(12 B_{0}\right)^{1 / p-1 / q}\left(\int_{9 B_{0}}|f|^{q} d \mu\right)^{1 / q}
\end{aligned}
$$

We obtain the conclusion of the theorem.

Lemma 15. If $\alpha \in(0,1), 1 \leq r<v \leq u<\infty, r<1 / \alpha$, and $1<u<1 / \alpha$, then

$$
\left|M_{r, \rho}^{\alpha} f(x)\right| \leq c\|f\|_{M_{v}^{u}(\mu)}^{u \alpha}\left|M_{r, \rho} f(x)\right|^{1-u \alpha} .
$$

Proof. This Proof is an analogy of $[18,29]$. For every $x \in \mathscr{X}$, we write $l_{x}^{1 / u}=\|f\|_{M_{v}^{u}(\mu)} / M_{r, \rho} f(x)$. So

$$
\begin{aligned}
\left|M_{r, \rho}^{\alpha} f(x)\right| \leq & \sup _{x \in B, \mu(\rho B) \leq l_{x}}\left[\frac{1}{\mu(\rho B)^{1-\alpha r}} \int_{B}|f|^{r} d \mu\right]^{1 / r} \\
& +\sup _{x \in B, \mu(\rho B)>i_{x}}\left[\frac{1}{\mu(\rho B)^{1-\alpha r}} \int_{B}|f|^{r} d \mu\right]^{1 / r} \\
= & I+I I .
\end{aligned}
$$

For $I$, we have

$$
\begin{aligned}
I & \leq \sup _{x \in B, \mu(\rho B) \leq l_{x}} \mu(\rho B)^{\alpha} \mu(\rho B)^{-1 / r}\left[\int_{B}|f|^{r} d \mu\right]^{1 / r} \\
& \leq l_{x}^{\alpha} M_{r, \rho} f(x) \\
& =\|f\|_{M_{v}^{u}(\mu)}^{u \alpha}\left|M_{r, \rho} f(x)\right|^{1-u \alpha} .
\end{aligned}
$$

If $\mu(\rho B)>l_{x}$, there exists a $i \in \mathbb{N}$ such that $2^{i-1} l_{x} \leq \mu(\rho B) \leq$ $2^{i} l_{x}$. It follows that

$$
\begin{aligned}
I I & \leq \sup _{x \in B, \mu(\rho B) \geq l_{x}}\left(2^{i-1} l_{x}\right)^{\alpha-1 / r}\left(\int_{B}|f|^{r} d \mu\right)^{1 / r} \\
& \leq \sup _{x \in B, \mu(\rho B) \geq l_{x}}\left(2^{i-1} l_{x}\right)^{\alpha-1 / r} \mu(B)^{1 / r-1 / v}\left(\int_{B}|f|^{v} d \mu\right)^{1 / v} \\
& \leq c\|f\|_{M_{v}^{u}(\mu)} \sup _{x \in B, \mu(\rho B) \geq l_{x}}\left(2^{i-1} l_{x}\right)^{\alpha-1 / r} \mu(\rho B)^{1 / r-1 / u} \\
& \leq c\|f\|_{M_{v}^{u}(\mu)} \sup _{i \in \mathbb{N}}\left(2^{i-1} l_{x}\right)^{\alpha-1 / r}\left(2^{i} l_{x}\right)^{1 / r-1 / u} \\
& \leq c\|f\|_{M_{v}^{u}(\mu)} \sup _{i \in \mathbb{N}}\left(2^{i}\right)^{\alpha-1 / u} l_{x}^{\alpha-1 / u} \\
& \leq c\|f\|_{M_{v}^{u}(\mu)}^{u \alpha}\left|M_{r, \rho} f(x)\right|^{1-u \alpha} .
\end{aligned}
$$

We complete the proof of Lemma 15.

Using Lemma 15 and Theorem 14, we have the following theorem.

Theorem 16. If $1<s \leq t<\infty, 1<r<u \leq v<1 / \alpha<\infty$, $\alpha=1 / u-1 / s$, and $s / t=u / v$, then operator $M_{r, \rho}^{\alpha}$ is bounded from $M_{v}^{u}(\mu)$ to $M_{t}^{s}(\mu)$.

\section{Fractional Integral Operator}

In this section, we prove the boundedness of fractional integral operator on Morrey space. The definition of fractional integral operator can be seen in [22]. The investigation of fractional integrals on quasimetric measure spaces with nondoubling measure (nonhomogeneous spaces) in Lebesgue spaces was researched in [30, chapter 6]. 
Definition 17. Let $0<\alpha<1$, for all $f \in L^{\infty}(\mu)$ with bounded support, as

$$
I_{\alpha} f(x)=\int_{X} \frac{f(y)}{\lambda(y, d(x, y))^{1-\alpha}} d \mu(y)
$$

In what follows, we assume that the dominating function $\lambda$ satisfies

$$
\lambda(x, a r)=a^{m} \lambda(x, r) \quad \forall x \in \mathscr{X}, a, r \in(0, \infty),
$$

where $\lambda$ is the dominating function of the measure of $\mu$ in Definition 3. The condition about $\lambda$ was first introduced by Bui and Duong in [11] to study the boundedness of commutators of Calderón-Zygmund operators. In [22], the authors obtain the boundedness of $I_{\alpha}$. The boundedness of fractional integral operators of other type can be seen in $[31,32]$.

Lemma 18. Let $\alpha \in(0,1), 1<p<1 / \alpha$, and $1 / q=1 / p-\alpha$. Then $I_{\alpha}$ is bounded from $L^{p}(\mu)$ space to $L^{q}(\mu)$ space.

Lemma 19. Let $\alpha \in(0,1), 1<q \leq p<1 / \alpha$, and $1 / t=$ $1 / p-\alpha$. Then

$$
\left|I_{\alpha} f(x)\right| \leq c\|f\|_{M_{q}^{p}(\mu)}^{1-p / t}\left(M_{6} f(x)\right)^{p / t}
$$

Proof. Let $s \in(0, \infty)$. We write

$$
\begin{aligned}
\left|I_{\alpha} f(x)\right| \leq & \int_{B(x, s)} \frac{|f(y)|}{\lambda(y, d(x, y))^{1-\alpha}} d \mu(y) \\
& +\int_{\mathscr{X}-B(x, s)} \frac{|f(y)|}{\lambda(y, d(x, y))^{1-\alpha}} d \mu(y) \\
= & I+I I .
\end{aligned}
$$

For $I$, we have

$$
\begin{gathered}
I \leq \sum_{j=0}^{\infty} \int_{B\left(x, 6^{-j} s\right)-B\left(x, 6^{-j-1} s\right)} \frac{|f(y)|}{\lambda(y, d(x, y))^{1-\alpha}} d \mu(y) \\
\leq \sum_{j=0}^{\infty} \frac{1}{\lambda\left(x, 6^{-j-1} s\right)^{1-\alpha}} \int_{B\left(x, 6^{-j} s\right)}|f(y)| d \mu(y) \\
\leq \sum_{j=0}^{\infty} \frac{\mu\left(B\left(x, 6^{-j+1} s\right)\right)}{\lambda\left(x, 6^{-j-1} s\right)^{1-\alpha}} \frac{1}{\mu\left(B\left(x, 6^{-j+1} s\right)\right)} \\
\quad \times \int_{B\left(x, 6^{-j} s\right)}|f(y)| d \mu(y)
\end{gathered}
$$

$$
\begin{aligned}
& \leq M_{6} f(x) \sum_{j=0}^{\infty} \frac{\lambda\left(x, 6^{-j+1} s\right)}{\lambda\left(x, 6^{-j-1} s\right)^{1-\alpha}} \\
& \leq M_{6} f(x) \sum_{j=0}^{\infty} \frac{\left(6^{-j+1} s\right)^{m} \lambda(x, 1)}{\left(6^{-j-1} s\right)^{m(1-\alpha)} \lambda(x, 1)^{1-\alpha}} \\
& \leq c M_{6} f(x) \sum_{j=0}^{\infty}\left(6^{m \alpha}\right)^{-j} \lambda(x, 1)^{\alpha} s^{m \alpha} \\
& \leq c M_{6} f(x) \lambda(x, 1)^{\alpha} s^{m \alpha} .
\end{aligned}
$$

Similarly, we have

$$
\begin{aligned}
I I & \leq \sum_{j=1}^{\infty} \int_{B\left(x, 6^{j} s\right)-B\left(x, 6^{j-1} s\right)} \frac{|f(y)|}{\lambda(y, d(x, y))^{1-\alpha}} d \mu(y) \\
\leq & \sum_{j=1}^{\infty} \frac{1}{\lambda\left(x, 6^{j-1} s\right)^{1-\alpha}} \int_{B\left(x, 6^{j} s\right)}|f(y)| d \mu(y) \\
\leq & \sum_{j=1}^{\infty} \frac{\mu\left(B\left(x, 6^{j} s\right)\right)^{1-1 / q}}{\lambda\left(x, 6^{j-1} s\right)^{1-\alpha}} \frac{\mu\left(B\left(x, 6^{j+1} s\right)\right)^{1 / p-1 / q}}{\mu\left(B\left(x, 6^{j+1} s\right)\right)^{1 / p-1 / q}} \\
& \times\left(\int_{B\left(x, 6^{j} s\right)}|f(y)|^{q} d \mu(y)\right)^{1 / q} \\
\leq & \|f\|_{M_{q}^{p}(\mu)} \sum_{j=1}^{\infty} \frac{\lambda\left(x, 6^{j+1} s\right)^{1-1 / p}}{\lambda\left(x, 6^{j-1} s\right)^{1-\alpha}} \\
\leq & c\|f\|_{M_{q}^{p}(\mu)} \lambda(x, 1)^{\alpha-1 / p}\left(s^{m}\right)^{\alpha-1 / p} .
\end{aligned}
$$

For every $x \in \mathscr{X}$, we take $s$ that satisfies $\lambda(x, 1) s^{m}=$ $\left(\|f\|_{M_{q}^{p}(\mu)} / M_{6} f(x)\right)^{p}$. Then

$$
\begin{aligned}
I & \leq c\|f\|_{M_{q}^{p}(\mu)}^{p \alpha} M_{6} f(x)^{1-p \alpha} \\
& \leq c\|f\|_{M_{q}^{p}(\mu)}^{1-p / t} M_{6} f(x)^{p / t}, \\
I I & \leq c\|f\|_{M_{q}^{p}(\mu)}^{1-p / t} M_{6} f(x)^{p / t} .
\end{aligned}
$$

So we have

$$
\left|I_{\alpha} f(x)\right| \leq c\|f\|_{M_{q}^{p}(\mu)}^{1-p / t}\left(M_{6} f(x)\right)^{p / t} .
$$

Using this lemma and the boundedness of maximal operator, we obtain the following result.

The following proof of Theorem 20 is similar to that of [33].

Theorem 20. Let $1<q \leq p<\infty, 1<t \leq s<\infty, \alpha \in(0,1)$, and $1 / s=1 / p-\alpha, s / t=p / q$. Then $I_{\alpha}$ is bounded from $M_{q}^{p}(\mu)$ space to $M_{t}^{s}(\mu)$ space. 
Proof. For all ball $B(x, r)$, we have

$$
\begin{aligned}
& \mu(2 B)^{t / s-1} \int_{B}\left|I_{\alpha} f\right|^{t} d \mu \\
& \leq c \mu(2 B)^{t / s-1} \int_{B}\|f\|_{M_{q}^{p}(\mu)}^{t-t p / s}\left(M_{6} f(x)\right)^{t p / s} d \mu \\
& \leq c \mu(2 B)^{t / s-1}\|f\|_{M_{q}^{p}(\mu)}^{t-q} \int_{B}\left(M_{6} f(x)\right)^{q} d \mu \\
& \leq c\|f\|_{M_{q}^{p}(\mu)}^{t} .
\end{aligned}
$$

Thus we have proved the theorem.

\section{Marcinkiewicz Integral Operator}

Firstly, we introduce the definition of Marcinkiewicz integral operator (see [23]).

Definition 21. Let $K$ be a locally integrable function on $(\mathscr{X} \times$ $\mathscr{X}-\{(x, x): x \in \mathscr{X}\})$. Assume that there exists a positive constant $c$ such that, for all $x, y, z \in \mathscr{X}$ with $x \neq y$,

$$
\begin{gathered}
|K(x, y)| \leq c \frac{d(x, y)}{\lambda(x, d(x, y))} \\
\int_{d(x, y) \geq 2 d(y, z)}[|K(x, y)-K(x, z)|+|K(y, x)-K(z, x)|] \\
\times \frac{1}{d(x, y)} d \mu(x) \leq c .
\end{gathered}
$$

The Marcinkiewicz integral $\mathscr{M}(f)$ associated with the above kernel $K$ is defined by setting

$$
\begin{aligned}
& \mathscr{M}(f)(x) \\
& =\left[\int_{0}^{\infty}\left|\int_{d(x, y)<t} K(x, y) f(y) d \mu(y)\right|^{2} \frac{d t}{t^{3}}\right]^{1 / 2} \quad \forall x \in \mathscr{X} .
\end{aligned}
$$

The boundedness on $L^{p}(\mu)$ has been proved in [23].

Lemma 22. Suppose that $\mathscr{M}$ is bounded on $L^{p_{0}}(\mu)$ space for some $p_{0} \in(1, \infty)$. Then $\mathscr{M}$ is bounded on $L^{p}(\mu)$ spaces for all $p \in(1, \infty)$.

Now we extend this result to the Morrey spaces $M_{q}^{p}(\mu)$.

Theorem 23. Let $1<p \leq q<\infty$. If $\mathscr{M}$ is bounded on $L^{p_{0}}(\mu)$ space for some $p_{0} \in(1, \infty)$, then $\mathscr{M}$ is bounded on $M_{q}^{p}(\mu)$ space.

Proof. For every ball $B=B\left(x_{0}, r\right), f \in M_{q}^{p}(\mu)$, let $f(x)=$ $f_{1}(x)+f_{2}(x)$, where $f_{1}(x)=f(x) \chi_{2 B}(x)$.
We can estimate

$$
\begin{aligned}
\mu(4 B)^{1 / p-1 / q}\left(\int_{B}|\mathscr{M}(f)|^{q} d \mu\right)^{1 / q} \\
\leq \mu(4 B)^{1 / p-1 / q}\left(\int_{B}\left|\mathscr{M}\left(f_{1}\right)\right|^{q} d \mu\right)^{1 / q} \\
\quad+\mu(4 B)^{1 / p-1 / q}\left(\int_{B}\left|\mathscr{M}\left(f_{2}\right)\right|^{q} d \mu\right)^{1 / q} \\
\leq I+I I .
\end{aligned}
$$

For the first term $I$, we have

$$
\begin{aligned}
I & \leq \mu(4 B)^{1 / p-1 / q}\left(\int_{\mathscr{X}}\left|f_{1}\right|^{q} d \mu\right)^{1 / q} \\
& \leq \mu(4 B)^{1 / p-1 / q}\left(\int_{2 B}|f|^{q} d \mu\right)^{1 / q} \leq\|f\|_{M_{q}^{p}(\mu)} .
\end{aligned}
$$

For $I I$, we firstly estimate $\mathscr{M}\left(f_{2}\right)(x)$, as

$$
\begin{aligned}
& \mathscr{M}\left(f_{2}\right)(x) \\
& \left.=\left[\int_{0}^{\infty}\left|\int_{d(x, y)<t} K(x, y) f_{2}(y) d \mu(y)\right|^{2} \frac{d t}{t^{3}}\right]^{1 / 2}\left|\int_{d(x, y)<t} K(x, y) f_{2}(y) d \mu(y)\right|^{2} \frac{d t}{t^{3}}\right]^{1 / 2} \\
& \leq\left[\int_{0}^{d\left(x_{0}, y\right)+2 r}\left|\int_{d(x, y)<t} K(x, y) f_{2}(y) d \mu(y)\right|^{2} \frac{d t}{t^{3}}\right]^{1 / 2} \\
& +\left[\int_{d\left(x_{0}, y\right)+2 r}^{\infty} \mid \int_{1}\right. \\
& \leq I I_{1}+I I_{2} .
\end{aligned}
$$

For any ball $B\left(x_{0}, r\right), y \in(k B)^{c}, k \geq 2$, and $x \in B$, we have

$$
\begin{aligned}
& \lambda\left(x_{0}, d\left(y, x_{0}\right)\right) \sim \lambda\left(y, d\left(y, x_{0}\right)\right) \sim \lambda(x, d(x, y)) \\
& I I_{1} \leq \int_{\mathscr{X}} K(x, y)\left|f_{2}(y)\right|\left[\int_{d(x, y)}^{d\left(x_{0}, y\right)+2 r} \frac{1}{t^{3}} d t\right]^{1 / 2} d \mu(y) \\
& \quad \leq \int_{\mathscr{X}} K(x, y)\left|f_{2}(y)\right|\left[\frac{1}{\left(d\left(x_{0}, y\right)+2 r\right)^{2}}-\frac{1}{d(x, y)^{2}}\right]^{1 / 2} d \mu(y) \\
& \quad \leq c \int_{X} K(x, y)\left|f_{2}(y)\right|\left[\frac{r}{\left(d\left(x_{0}, y\right)\right)^{3}}\right]^{1 / 2} d \mu(y) \\
& \quad \leq c \int_{X} \frac{d(x, y)}{\lambda(x, d(x, y))}\left|f_{2}(y)\right|\left[\frac{r}{\left(d\left(x_{0}, y\right)\right)^{3}}\right]^{1 / 2} d \mu(y) \\
& \quad \leq c \int_{X-2 B} \frac{r^{1 / 2}}{d\left(x_{0}, y\right)^{1 / 2} \lambda\left(x_{0}, d\left(x_{0}, y\right)\right)}\left|f_{2}(y)\right| d \mu(y)
\end{aligned}
$$




$$
\begin{aligned}
& \leq c r^{1 / 2} \sum_{i=2}^{\infty} \int_{B\left(x_{0}, 2^{i} r\right)-B\left(x_{0}, 2^{i-1} r\right)} \frac{|f(y)|}{d\left(x_{0}, y\right)^{1 / 2} \lambda\left(x_{0}, d\left(x_{0}, y\right)\right)} d \mu(y) \\
& \leq c r^{1 / 2} \sum_{i=2}^{\infty} \frac{1}{\lambda\left(x_{0}, 2^{i-1} r\right)\left(2^{i-1} r\right)^{1 / 2}} \int_{B\left(x_{0}, 2^{i} r\right)}|f(y)| d \mu(y) \\
& \leq c\|f\|_{M_{q}^{p}(\mu)} \sum_{i=2}^{\infty} \frac{\mu\left(B\left(x_{0}, 2^{i+1} r\right)\right)^{1-1 / p}}{\lambda\left(x_{0}, 2^{i-1} r\right)} \\
& \leq c\|f\|_{M_{q}^{p}(\mu)} \sum_{i=2}^{\infty} \frac{\left(2^{i+1} r\right)^{m-m / p} \lambda\left(x_{0}, 1\right)^{1-1 / p}}{\left(2^{i-1} r\right)^{m} \lambda\left(x_{0}, 1\right)} \\
& \leq c\|f\|_{M_{q}^{p}(\mu)} r^{m(-1 / p)} \lambda(x, 1)^{-1 / p} \leq c\|f\|_{M_{q}^{p}(\mu)} \lambda\left(x_{0}, r\right)^{-1 / p} .
\end{aligned}
$$

Similarly, we obtain

$$
\begin{aligned}
I I_{2} & \leq c \int_{\mathscr{X}-2 B} \frac{1}{\lambda\left(x_{0}, d\left(x_{0}, y\right)\right)}|f(y)| d \mu(y) \\
& \leq c \sum_{i=2}^{\infty} \int_{B\left(x_{0}, 2^{i} r\right)-B\left(x_{0}, 2^{i-1} r\right)} \frac{|f(y)|}{\lambda\left(x_{0}, d\left(x_{0}, y\right)\right)} d \mu(y) \\
& \leq c \sum_{i=2}^{\infty} \frac{1}{\lambda\left(x_{0}, 2^{i-1} r\right)} \int_{B\left(x_{0}, 2^{i} r\right)}|f(y)| d \mu(y) \\
& \leq c\|f\|_{M_{q}^{p}(\mu)} \sum_{i=2}^{\infty} \frac{\mu\left(B\left(x_{0}, 2^{i+1} r\right)\right)^{1-1 / p}}{\lambda\left(x_{0}, 2^{i-1} r\right)} \\
& \leq c\|f\|_{M_{q}^{p}(\mu)} \lambda\left(x_{0}, r\right)^{-1 / p} .
\end{aligned}
$$

That is to say, $\mathscr{M}\left(f_{2}\right)(x) \leq c \lambda\left(x_{0}, r\right)^{-1 / p}\|f\|_{M_{q}^{p}(\mu)}$ for all ball $B\left(x_{0}, r\right)$ and $x \in B\left(x_{0}, r\right)$.

Using it we have

$$
\begin{aligned}
I I & \leq \mu(4 B)^{1 / p-1 / q}\left(\int_{B}\left|\mathcal{M}\left(f_{2}\right)\right|^{q} d \mu\right)^{1 / q} \\
& \leq c \mu(4 B)^{1 / p-1 / q}\left(\int_{B}\left|\lambda\left(x_{0}, r\right)^{-1 / p}\|f\|_{M_{q}^{p}(\mu)}\right|^{q} d \mu\right)^{1 / q} \\
& \leq c\|f\|_{M_{q}^{p}(\mu)} \mu(4 B)^{1 / p-1 / q} \lambda\left(x_{0}, r\right)^{-1 / p} \mu(B)^{1 / q} \\
& \leq c\|f\|_{M_{q}^{p}(\mu)} \mu(B)^{1 / p} \lambda\left(x_{0}, r\right)^{-1 / p} \leq c\|f\|_{M_{q}^{p}(\mu)} .
\end{aligned}
$$

The proof of Theorem 23 is completed.

\section{Acknowledgments}

Cao Yonghui is supported by the National Natural Science Foundation of China (Grant no. 11261055) and by the National Natural Science Foundation of Xinjiang (Grant no. 2011211A005). Zhou Jiang is supported by the National Science Foundation of China (Grant nos. 11261055 and 11161044) and the National Natural Science Foundation of Xinjiang (Grant nos. 2011211A005 and BS120104).

\section{References}

[1] J. García-Cuerva and A. E. Gatto, "Boundedness properties of fractional integral operators associated to non-doubling measures," Studia Mathematica, vol. 162, no. 3, pp. 245-261, 2004.

[2] G. Hu, Y. Meng, and D. Yang, "Multilinear commutators for fractional integrals in non-homogeneous spaces," Publicacions Matemàtiques, vol. 48, no. 2, pp. 335-367, 2004.

[3] X. Tolsa, "BMO, $H^{1}$, and Calderón-Zygmund operators for non doubling measures," Mathematische Annalen, vol. 319, no. 1, pp. 89-149, 2001.

[4] X. Tolsa, "Littlewood-Paley theory and the T(1) theorem with non-doubling measures," Advances in Mathematics, vol. 164, no. 1, pp. 57-116, 2001.

[5] X. Tolsa, "The space $H^{1}$ for nondoubling measures in terms of a grand maximal operator," Transactions of the American Mathematical Society, vol. 355, no. 1, pp. 315-348, 2003.

[6] X. Tolsa, "Painlevé's problem and the semiadditivity of analytic capacity," Acta Mathematica, vol. 190, no. 1, pp. 105-149, 2003.

[7] X. Tolsa, "The semiadditivity of continuous analytic capacity and the inner boundary conjecture," American Journal of Mathematics, vol. 126, no. 3, pp. 523-567, 2004.

[8] W. Chen, Y. Meng, and D. Yang, "Calderón-Zygmund operators on Hardy spaces without the doubling condition," Proceedings of the American Mathematical Society, vol. 133, no. 9, pp. 26712680, 2005.

[9] W. Chen and E. Sawyer, "A note on commutators of fractional integrals with $(u)$ functions," Illinois Journal of Mathematics, vol. 46, no. 4, pp. 1287-1298, 2002.

[10] T. Hytönen, "A framework for non-homogeneous analysis on metric spaces, and the RBMO space of Tolsa," Publicacions Matemàtiques, vol. 54, no. 2, pp. 485-504, 2010.

[11] T. A. Bui and X. T. Duong, "Hardy spaces, regularized BMO spaces and the boundedness of Calderón-Zygmund operators on non-homogeneous spaces," Journal of Geometric Analysis, vol. 23, no. 2, pp. 895-932, 2013.

[12] T. Hytönen, S. Liu, D. Yang, and D. Yang, "Boundedness of Calderón-Zygmund operators on non-homogeneous metric measure spaces," Canadian Journal of Mathematics, vol. 64, no. 4, pp. 892-923, 2012.

[13] J. Zhou and B. L. Ma, "Marcinkiewicz commutators with Lipschitz functions in non-homogeneous spaces," Canadian Mathematical Bulletin, vol. 55, no. 3, pp. 646-662, 2012.

[14] T. Hytönen, D. Yang, and D. Yang, "The Hardy space $H^{1}$ on nonhomogeneous metric spaces," Mathematical Proceedings of the Cambridge Philosophical Society, vol. 153, no. 1, pp. 9-31, 2012.

[15] H. Lin, E. Nakai, and D. Yang, "Boundedness of Lusin-area and $g_{\lambda}^{*}$ functions on localized BMO spaces over doubling metric measure spaces," Bulletin des Sciences Mathématiques, vol. 135, no. 1, pp. 59-88, 2011.

[16] H. Lin and D. Yang, "An interpolation theorem for sublinear operators on non-homogeneous metric measure spaces," Banach Journal of Mathematical Analysis, vol. 6, no. 2, pp. 168179, 2012.

[17] S. Liu, D. Yang, and D. Yang, "Boundedness of CalderónZygmund operators on non-homogeneous metric measure spaces: equivalent characterizations," Journal of Mathematical Analysis and Applications, vol. 386, no. 1, pp. 258-272, 2012.

[18] Y. Sawano and H. Tanaka, "Morrey spaces for non-doubling measures," Acta Mathematica Sinica, vol. 21, no. 6, pp. 15351544, 2005. 
[19] Y. Sawano, " $l^{q}$-valued extension of the fractional maximal operators for non-doubling measures via potential operators," International Journal of Pure and Applied Mathematics, vol. 26, no. 4, pp. 505-523, 2006.

[20] Y. Sawano and H. Tanaka, "Equivalent norms for the Morrey spaces with non-doubling measures," Far East Journal of Mathematical Sciences, vol. 22, no. 3, pp. 387-404, 2006.

[21] F. Chiarenza and M. Frasca, "Morrey spaces and HardyLittlewood maximal function," Rendiconti di Matematica e delle sue Applicazioni, vol. 7, no. 3-4, pp. 273-279, 1987.

[22] X. Fu, D. Yang, and W. Yuan, "Generalized fractional integrals and their commutators over non-homogeneous spaces," 2012.

[23] H. Lin and D. Yang, "Equivalent Boundedness of Marcinkiewicz integrals on nonhomogeneous metric measure spaces," 2012.

[24] V. S. Guliyev and Y. Sawano, "Linear and sublinear operators on Generalized Morrey spaces with non-doubling measures," Publicationes Mathematicae Debrecen, vol. 83, no. 3, pp. 1-17, 2013.

[25] J. Heinonen, Lectures on Analysis on Metric Spaces, Springer, New York, NY, USA, 2001.

[26] G. Di Fazio and M. A. Ragusa, "Commutators and Morrey spaces," Unione Matematica Italiana. Bollettino A, vol. 5, no. 3, pp. 323-332, 1991.

[27] J. Peetre, "On the theory of $L_{p}^{\lambda}$ spaces," Journal of Functional Analysis, vol. 4, pp. 71-87, 1969.

[28] V. Kokilashvili and A. Meskhi, "Maximal functions and potentials in variable exponent Morrey spaces with non-doubling measure," Complex Variables and Elliptic Equations, vol. 55, no. 8-10, pp. 923-936, 2010.

[29] L. I. Hedberg, "On certain convolution inequalities," Proceedings of the American Mathematical Society, vol. 36, pp. 505-510, 1972.

[30] D. E. Edmunds, V. Kokilashvili, and A. Meskhi, Bounded and Compact Integral Operators, chapter 6, Kluwer Academic Publishers, Dordrecht, The Netherlands, 2002.

[31] I. Sihwaninggrum and Y. Sawano, "Weak and strong type estimates for fractional integral opertors on Morrey spaces in metric measure spaces," Eurasian Mathematical Journal, vol. 4, no. 1, pp. 76-81, 2013.

[32] A. Eridani, V. Kokilashvili, and A. Meskhi, "Morrey spaces and fractional integral operators," Expositiones Mathematicae, vol. 27, no. 3, pp. 227-239, 2009.

[33] D. R. Adams, "A note on Riesz potentials," Duke Mathematical Journal, vol. 42, no. 4, pp. 765-778, 1975. 


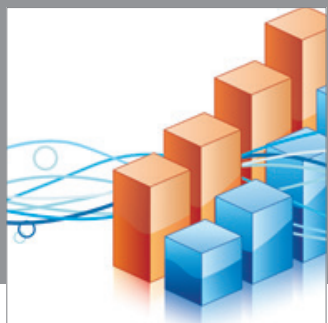

Advances in

Operations Research

mansans

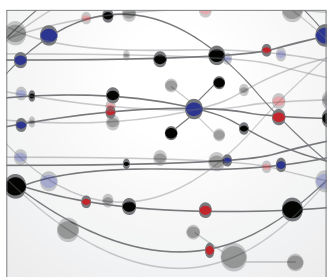

The Scientific World Journal
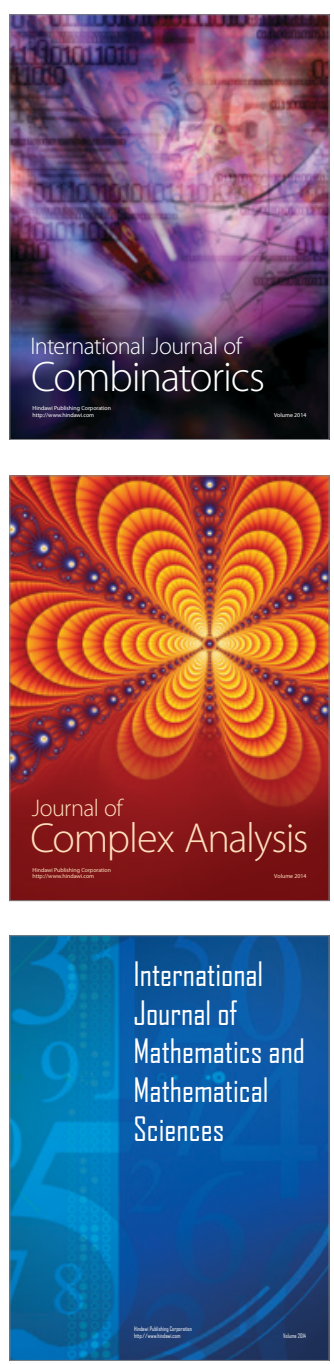
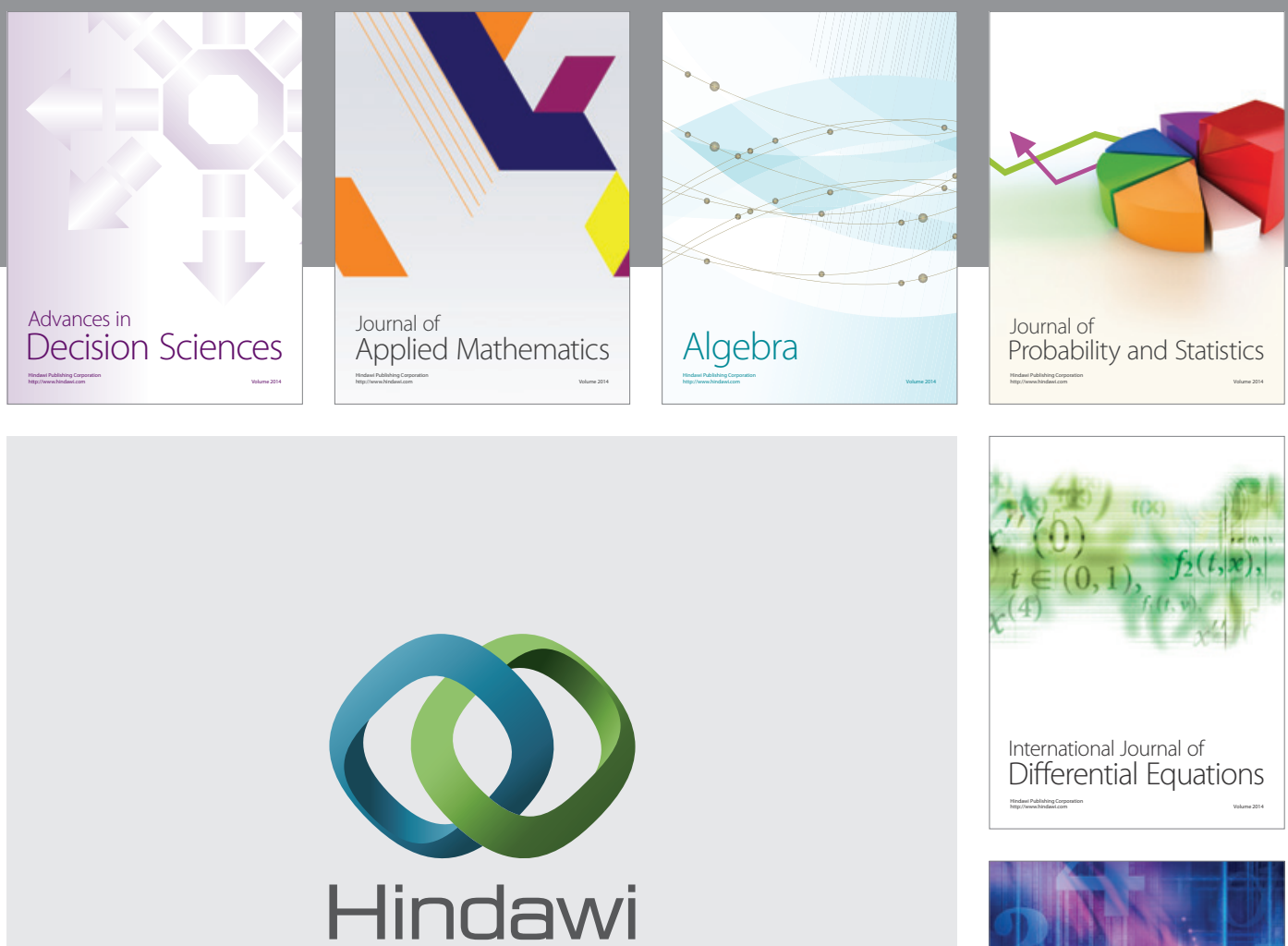

Submit your manuscripts at http://www.hindawi.com
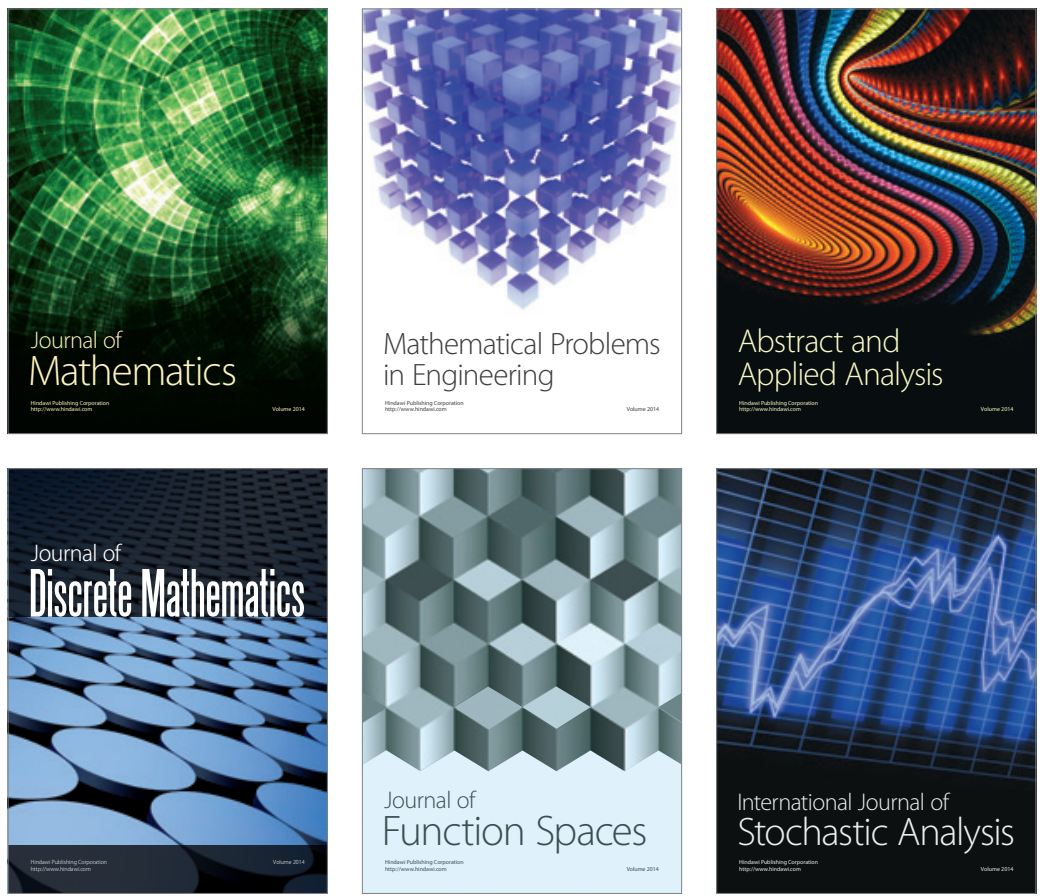

Journal of

Function Spaces

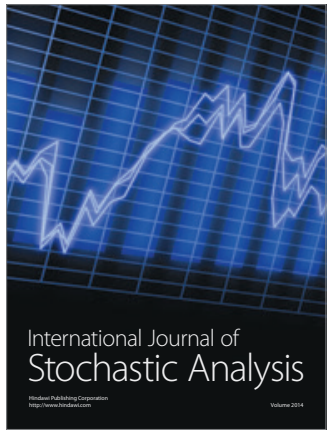

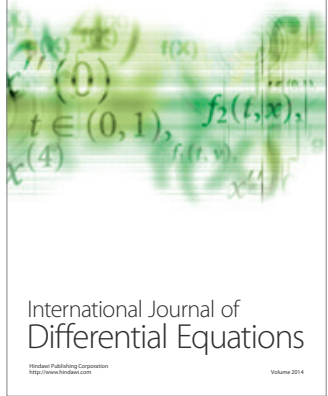
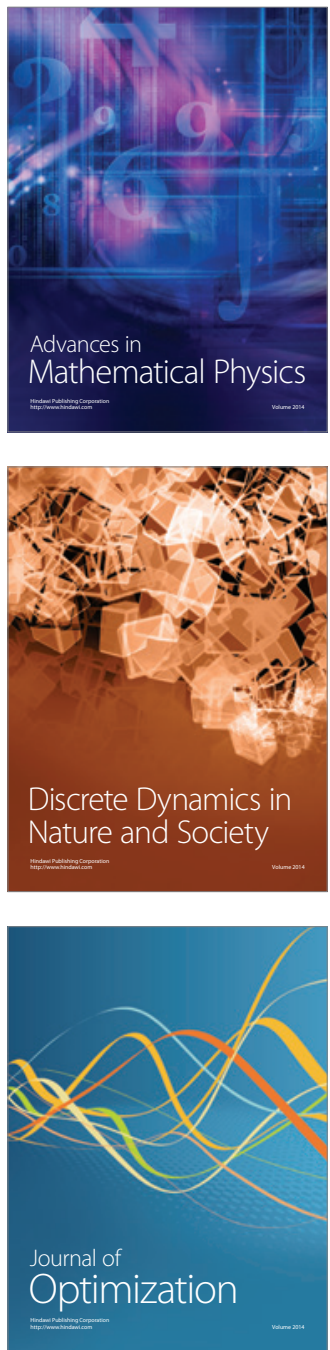